\title{
Multi-level Dynamic Guard Channels for Priority Access in Cellular Systems
}

\author{
Tung Chong Wong ${ }^{1}$, Jon W. Mark ${ }^{2}$, and Kee Chaing Chua ${ }^{3}$ \\ ${ }^{1}$ Institute for Infocomm Research, 21 Heng Mui Keng Terrace, \\ Singapore 119613, Singapore \\ wongtc@i2r.a-star.edu.sg \\ ${ }^{2}$ Center for Wireless Communications, University of Waterloo, \\ Waterloo, Ontario, Canada N2L 3G1 \\ jwmark@bbcr. uwaterloo.ca \\ ${ }^{3}$ Electrical and Computer Engineering, National University of Singapore, \\ 10 Kent Ridge Crescent, Singapore 119260, Singapore \\ chuakc@nus.edu.sg
}

\begin{abstract}
A multi-level dynamic guard channels (MLDGC) scheme to efficiently provide priority access for handoff calls over new calls in cellular systems is proposed. The switch between levels is controlled by the number of dropped calls. Each additional level has an increasing number of guard channels as triggered by the number of dropped calls. An analytical formulation of the steady state probabilities, new call blocking probability, handoff call blocking probability and system utilization is presented. The handoff call arrival rate is iteratively obtained. Numerical results show that the performance of MLDGC is almost the same as a fixed guard channels (FGC) scheme under light load. However, the advantage of the MLDGC scheme is demonstrated under heavy load where the handoff call blocking probability is much better than that of a FGC scheme, giving more priority and protection to handoff calls over new calls.
\end{abstract}

\section{Introduction}

In mobile communications, efficient handoff to minimize the number of handoff call dropping is critically important. One of the most fundamental approaches to provide access for handoff calls over new calls is the fixed guard channels (FGC) scheme [1]. By reserving a small number of guard channels for the exclusive use by handoff calls, the handoff call blocking probability is significantly reduced at the expense of a slight increase in the new call blocking probability. Thus priority is given to handoff calls over new calls as new call blockings are more acceptable to users than handoff call blocking. That is, once a call is admitted, a user is more likely to object to being dropped in the middle of a call than when a user cannot get into the system at the start of a new call.

Dynamic guard channels (DGC) schemes have been proposed and studied [2-6]. Refs. [2-3] consider a DGC scheme which adapts the number of guard channels in each radio cell according to the current estimate of the handoff call arrival rate. This 
rate is derived based on the current number of ongoing calls in the neighbouring cells and the mobility pattern. A DGC scheme which adapts to the traffic load is proposed in [4]. Another DGC scheme which varies the number of guard channels based on the current number of users in a reservation cluster is proposed in [5]. Ref. [6] models a FGC scheme and a DGC scheme for mobile transactions. The performances of the DGC schemes in [2-4] are evaluated using computer simulations, while those in [5-6] are evaluated using numerical techniques.

In this paper, we propose a multi-level DGC (MLDGC) scheme. This MLDGC scheme has multiple levels of states that adapt to varying number of guard channels. Each additional level has an increasing number of guard channels as triggered by the number of dropped handoff calls. The greater the number of dropped handoff calls, the higher the level in the MLDGC scheme. The higher the level, the greater the number of guard channels. The advantage of MLDGC is that the handoff call blocking probability is much better than that of a FGC scheme under heavy traffic load, giving higher access priority and protection to handoff calls over new calls. An analytical formulation of the steady state probabilities, new call blocking probability, handoff call blocking probability and system utilization is presented in the sequel. The handoff call arrival rate is iteratively obtained.

The rest of the paper is organized as follows. Section 2 describes the system model, system level and call level parameters. Section 3 describes the proposed MLDGC scheme. Section 4 presents an analytical model for the MLDGC scheme. Numerical results for a two-level MLDGC scheme are presented in Section 5. Finally, concluding remarks are made in Section 6.

\section{System Model}

Without loss of generality, we consider a cellular system consisting of square cells. The following system parameters are used throughout the paper.

\section{System Level Parameters}

$C$ : total number of channels in a cell

$C_{G k}$ : number of guard channels in level $k$ of the MLDGC scheme in a cell, $k=1,2, \ldots, K$, and $C_{G 1}<C_{G 2}<\ldots<C_{G K}$

$K$ : number of levels in the MLDGC scheme

The call level parameters used throughout the paper are listed below.

\section{Call Level Parameters}

$B_{n}$ : new call blocking probability

$B_{h}$ : handoff call blocking probability

$N_{u}$ : system utilization

$\lambda_{n}:$ arrival rate of new calls 
$\lambda_{h}:$ arrival rate of handoff calls

$\lambda=\lambda_{n}+\lambda_{h}$ : mean call arrival rate of new and handoff calls

$\mu_{c}^{-1}:$ mean call holding time of a call in a cell

$\mu_{h}^{-1}:$ mean dwell time (interhandoff time) of a call in a cell

$\mu=\mu_{c}+\mu_{h}$ : mean equivalent service rate of a call in a cell

The dynamics of a radio cell is driven by new call requests, call terminations, and handoffs induced by user mobility. Maintaining an ongoing call is more important than admitting a new call. Hence, handoff calls should be given a higher access priority, or a lower blocking probability than new calls.

\section{Multi-level Dynamic Guard Channel (MLDGC) Scheme}

The MLDGC scheme works as follows. There are $K$ levels in the MLDGC scheme. In level 1, the MLDGC scheme works similarly to a FGC scheme. A small number of guard channels, $C_{G l}$, in a cell is reserved for exclusive use by handoff calls, while the other channels in the cell can be used by both the handoff calls and the new calls. However, the MLDGC scheme differs from the FGC scheme in that the former will be triggered to move to the next level (level 2) when a handoff call is dropped because there is no available channel in the cell. In level 2, more guard channels, $C_{G 2}$ $\left(>C_{G I}\right)$, are allocated for exclusive use by handoff calls than in level 1 . When these guard channels are no longer occupied by handoff calls, the scheme will switch back to level 1. Similarly, when another handoff call is dropped due to full channel occupancy while in level 2, the MLDGC scheme will move to the next level (level 3) with even more guard channels, $C_{G 3}\left(>C_{G 2}>C_{G 1}\right)$, allocated in this new level. Similarly, if these guard channels are no longer occupied by handoff calls, the scheme will switch back to level 1. This dynamic allocation of guard channels can continue up to a maximum of $K$ levels. At the $K$ th level, the number of guard channels is, $C_{G K}$, such that $C_{G K}>\ldots>C_{G 3}>C_{G 2}>C_{G 1}$.

Fig. 1 shows a two-dimensional finite state Markov chain for the MLDGC scheme. State $(k, i)$ represents the $k$ th level of the MLDGC scheme and there are $i$ channels in service in the cell. $\lambda\left(=\lambda_{n}+\lambda_{h}\right)$ is the mean call arrival rate of new and handoff calls in a cell, while $\lambda_{n}$ and $\lambda_{h}$ are respectively the arrival rate of new calls and handoff calls in the cell. $\mu\left(=\mu_{c}+\mu_{h}\right)$ is the mean equivalent service rate of a call in a cell, while $\mu_{c}^{-1}$ and $\mu_{h}^{-1}$ are respectively the mean call holding time of a call and the mean dwell time (interhandoff time) of a call in the cell. In level 1 of the MLDGC scheme, only handoff calls can be admitted in the $C_{G l}$ channels as indicated by the transition rates of $\lambda_{h}$ in Fig. 1. On the other hand, the rest of the channels can be used by both the new and handoff calls as indicated by the transition rates of $\lambda$. If the MLDGC scheme is in state $(k, C), k=1,2, \ldots K-1$, it will be triggered to jump to the state $(k+1, C)$ in the next level of $(k+1)$ by a handoff call arriving to a fully occupied cell. This transition is indicated by the state transition rate of $\lambda_{h}$ from state $(k, C)$ to state $(k+1, C)$. For every jump to the next level, the number of guard channels is increased such that $C_{G K}>\ldots>C_{G 3}>C_{G 2}>C_{G 1}$. Note that in levels 2 to $K$, only handoff calls can 
use the respective level's guard channels as indicated by the transition rates of $\lambda_{h}$. In state $(k, i)$, the channels are serviced at a mean rate of $i \mu$ and it will jump to state $(k, i$ 1) due to a call termination or a call handoff to another cell. If the MLDGC scheme is in state $\left(k, C-C_{G k}\right)$ in level $k, k=2,3, \ldots, K$, it will return to state $\left(1, C-C_{G k}-1\right)$ in level 1 with a transition rate of $\left(C-C_{G k}\right) \mu$ if a channel has been served due to call termination or call handoff to another cell before any handoff call arrival.

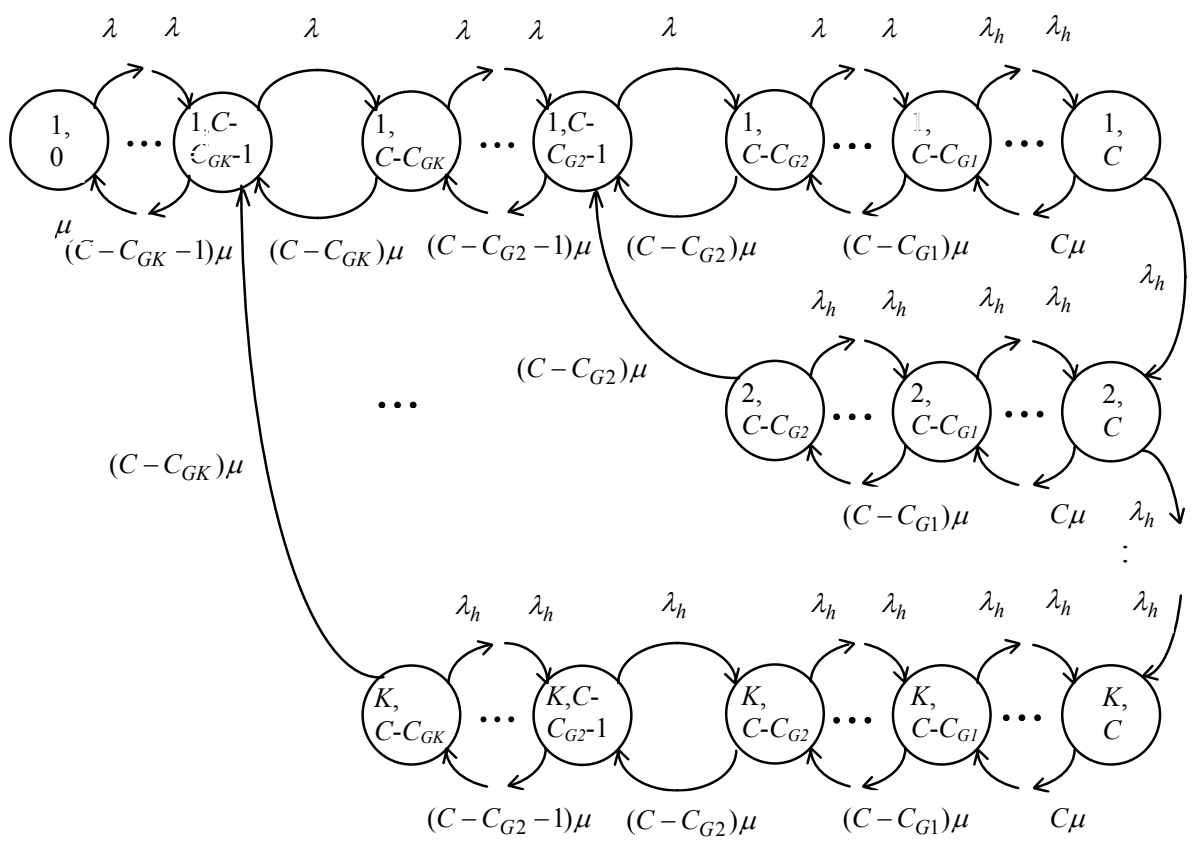

Fig. 1. State transition diagram for the MLDGC scheme

\section{Analytical Model}

Consider a 2-level MLDGC model, that is, $K=2$. The guard channels, $C_{G k}$, are reserved for handoff connections only. To facilitate analytical modeling, it is necessary to make certain assumptions about the traffic parameters. It is not unreasonable to assume that the holding time has a negative exponential distribution [7]. Although a negative exponential distribution assumption may not be as reasonable for the cell dwell time, for analytical tractability, we will make the same assumption for cell dwell time (interhandoff time) [7] and model the channel occupancy as a 2-dimensional Markov chain with the call level transition rate parameters as in Fig. 1. This Markov chain can be modeled and solved using numerical techniques. Instead of solving this Markov chain using numerical techniques, this Markov chain is solved analytically by equating probability flows in this section.

Let $P_{k, i}$ be the steady state probabilities in state $(k, i)$ of the Markov chain, where $k=1, \ldots, K$, and $i=0, \ldots, C$ for $\mathrm{k}=1$ and $i=C-C_{G k}, \ldots, C$ for $k=2, \ldots, K$. Solving the Markov 
chain in Fig. 1 by equating probability flows across surfaces of the Markov chain and using the sum of total state probabilities as one, we get

$$
\begin{aligned}
& P_{1, i}=P_{1,0}\left(\frac{\lambda}{\mu}\right)^{i} \frac{1}{i !}, \\
& P_{1, i}=P_{1,0} \frac{1}{X}\left(\frac{\lambda}{\mu}\right)^{C-C_{G 2}-1} \frac{1}{\left(C-C_{G 2}-1\right) !}\left[\frac{\lambda_{h}}{\lambda}+\frac{(C-j+1) \mu}{\lambda}\left[\frac{\lambda_{h}}{\lambda}+\frac{(C-j+2) \mu}{\lambda}[\ldots\right.\right. \\
& \times\left[\frac{\lambda_{h}}{\lambda}+\frac{\left(C-C_{G 1}\right) \mu}{\lambda}\left[1+\frac{\left(C-C_{G 1}+1\right) \mu}{\lambda_{h}}\left[1+\frac{\left(C-C_{G 1}+2\right) \mu}{\lambda_{h}}\left[\ldots \left[1+\frac{(C-1) \mu}{\lambda_{h}}\right.\right.\right.\right.\right. \\
& \left.\left.\left.\left.\left.\left.\left.\times\left[1+\frac{C \mu}{\lambda_{h}}\right]\right] \ldots\right]\right]\right]\right] \ldots\right]\right], \quad C-C_{G 2}-1 \leq i \leq C-C_{G 1}-1, j=C-i, \\
& P_{1, i}=P_{1,0} \frac{1}{X}\left(\frac{\lambda}{\mu}\right)^{C-C_{G 2}-1} \frac{1}{\left(C-C_{G 2}-1\right) !}\left[1+\frac{(C-j+1) \mu}{\lambda_{h}}\left[1+\frac{(C-j+2) \mu}{\lambda_{h}}[\ldots\right.\right. \\
& \left.\left.\left.\times\left[1+\frac{(C-1) \mu}{\lambda_{h}}\left[1+\frac{C \mu}{\lambda_{h}}\right]\right] \ldots\right]\right]\right], \quad C-C_{G 1} \leq i \leq C-1, j=C-i, \\
& P_{1, i}=P_{1,0} \frac{1}{X}\left(\frac{\lambda}{\mu}\right)^{C-C_{G 2}-1} \frac{1}{\left(C-C_{G 2}-1\right) !}, \\
& i=C, \\
& P_{2, i}=P_{1,0} \frac{1}{X}\left(\frac{\lambda_{h}}{\left(C-C_{G 2}\right) \mu}\right)\left(\frac{\lambda}{\mu}\right)^{C-C_{G 2}-1} \frac{1}{\left(C-C_{G 2}-1\right) !}, \quad i=C-C_{G 2}, \\
& P_{2, i}=P_{1,0} \frac{1}{X}\left(\frac{\lambda_{h}}{\left(C-C_{G 2}\right) \mu}\right)\left(\frac{\lambda}{\mu}\right)^{C-C_{G 2}-1} \frac{1}{\left(C-C_{G 2}-1\right) !}\left[\frac { C - C _ { G 2 } } { C - j } \left[1+\frac{\lambda_{h}}{(C-j-1) \mu}\right.\right. \\
& \times\left[1+\frac{\lambda_{h}}{(C-j-2) \mu}\left[\ldots \left[1+\frac{\lambda_{h}}{\left(C-C_{G 2}+2\right) \mu}\left[1+\frac{\lambda_{h}}{\left(C-C_{G 2}+1\right) \mu}\right.\right.\right.\right. \\
& \left.\left.\left.\left.\left.\times\left[1+\frac{\lambda_{h}}{\left(C-C_{G 2}\right) \mu}\right]\right]\right] \ldots\right]\right]\right], \quad C-C_{G 2}+1 \leq i \leq C, j=C-i \text {. }
\end{aligned}
$$

where

$$
\begin{aligned}
X & =\left[\frac{\lambda_{h}}{\lambda}+\frac{\left(C-C_{G 2}\right) \mu}{\lambda}\left[\frac{\lambda_{h}}{\lambda}+\frac{\left(C-C_{G 2}+1\right) \mu}{\lambda}\left[\ldots \left[\frac{\lambda_{h}}{\lambda}+\frac{\left(C-C_{G 1}\right) \mu}{\lambda}\right.\right.\right.\right. \\
& \times\left[1+\frac{\left(C-C_{G 1}+1\right) \mu}{\lambda_{h}}\left[1+\frac{\left(C-C_{G 1}+2\right) \mu}{\lambda_{h}}\left[\ldots \left[1+\frac{(C-1) \mu}{\lambda_{h}}\right.\right.\right.\right. \\
& \left.\left.\left.\left.\left.\times\left[1+\frac{C \mu}{\lambda_{h}}\right] \ldots\right]\right]\right] \ldots\right]\right],
\end{aligned}
$$


and

$$
\begin{aligned}
& P_{1,0}=\left\{1+\sum_{i=1}^{C-C_{G 2}-1}\left(\frac{\lambda}{\mu}\right)^{i} \frac{1}{i !}+\sum_{j=C_{G 1}+1}^{C_{G 2}} \frac{1}{X}\left(\frac{\lambda}{\mu}\right)^{C-C_{G 2}-1} \frac{1}{\left(C-C_{G 2}-1\right) !}\right. \\
& \times\left[\frac{\lambda_{h}}{\lambda}+\frac{(C-j+1) \mu}{\lambda}\left[\frac{\lambda_{h}}{\lambda}+\frac{(C-j+2) \mu}{\lambda}\left[\ldots \left[\frac{\lambda_{h}}{\lambda}+\frac{\left(C-C_{G 1}\right) \mu}{\lambda}\left[1+\frac{\left(C-C_{G 1}+1\right) \mu}{\lambda_{h}}\right.\right.\right.\right.\right. \\
& \left.\left.\left.\left.\left.\times\left[1+\frac{\left(C-C_{G 1}+2\right) \mu}{\lambda_{h}}\left[\ldots\left[1+\frac{(C-1) \mu}{\lambda_{h}}\left[1+\frac{C \mu}{\lambda_{h}}\right]\right] \ldots\right]\right]\right]\right]\right]\right]\right] \\
& +\sum_{j=1}^{C_{G 1}} \frac{1}{X}\left(\frac{\lambda}{\mu}\right)^{C-C_{G 2}-1} \frac{1}{\left(C-C_{G 2}-1\right) !}\left[1+\frac{(C-j+1) \mu}{\lambda_{h}}\left[1+\frac{(C-j+2) \mu}{\lambda_{h}}[. .\right.\right. \\
& \left.\left.\times\left[1+\frac{(C-1) \mu}{\lambda_{h}}\left[1+\frac{C \mu}{\lambda_{h}}\right]\right] \cdots\right]\right]+\frac{1}{X}\left(\frac{\lambda}{\mu}\right)^{C-C_{G 2}-1} \frac{1}{\left(C-C_{G 2}-1\right) !} \\
& +\frac{1}{X}\left(\frac{\lambda_{h}}{\left(C-C_{G 2}\right) \mu}\right)\left(\frac{\lambda}{\mu}\right)^{C-C_{G 2}-1} \frac{1}{\left(C-C_{G 2}-1\right) !}+\sum_{j=0}^{C_{G 2}-1} \frac{1}{X}\left(\frac{\lambda_{h}}{\left(C-C_{G 2}\right) \mu}\right) \\
& \times\left(\frac{\lambda}{\mu}\right)^{C-C_{G 2}-1} \frac{1}{\left(C-C_{G 2}-1\right) !}\left[\frac { C - C _ { G 2 } } { C - j } \left[1+\frac{\lambda_{h}}{(C-j-1) \mu}\left[1+\frac{\lambda_{h}}{(C-j-2) \mu}[\ldots\right.\right.\right. \\
& \left.\left.\left.\left.\times\left[1+\frac{\lambda_{h}}{\left(C-C_{G 2}+2\right) \mu}\left[1+\frac{\lambda_{h}}{\left(C-C_{G 2}+1\right) \mu}\left[1+\frac{\lambda_{h}}{\left(C-C_{G 2}\right) \mu}\right]\right]\right] \ldots\right]\right]\right]\right\}^{-1}
\end{aligned}
$$

The new call blocking probability, $B_{n}$, is given by

$$
B_{n}=\sum_{i=C-C_{G 1}}^{C} P_{1, i}+\sum_{i=C-C_{G 2}}^{C} P_{2, i} .
$$

The handoff call blocking probability, $B_{h}$, is given by

$$
B_{h}=P_{1, C}+P_{2, C}
$$

The system utilization, $N_{u}$, is given by

$$
N_{u}=\sum_{i=1}^{C} i P_{1, i}+\sum_{i=C-C_{G 2}}^{C} i P_{2, i}=\frac{\lambda_{n}\left(1-B_{n}\right)+\lambda_{h}\left(1-B_{h}\right)}{\mu} .
$$

Equating the handoff call arrival rate to the product of the average handoff rate for a call and the average number of calls, we have

$$
\lambda_{h}=\mu_{h} N_{u}=\mu_{h} \times \frac{\left(1-B_{n}\right) \lambda_{n}+\left(1-B_{h}\right) \lambda_{h}}{\mu_{c}+\mu_{h}}=\frac{\mu_{h}\left(1-B_{n}\right) \lambda_{n}}{\mu_{c}+B_{h} \mu_{h}} .
$$


Thus, the handoff call arrival rate can be approximated under low blocking probabilities as follows:

$$
\lambda_{h}=\mu_{h} \lambda_{n} / \mu_{c}
$$

where $\mu_{h}=v / s, v$ is the speed of the class $k$ mobile and $s$ is the cell length of a square cell.

Note that the steady state probabilities, $P_{k, i}$ 's, are functions of the handoff call arrival rate, $\lambda_{h}$, and $\lambda_{h}$ is a function of the system utilization, $N_{u}$, which is a function of $P_{k, i}$ 's. We use an iterative method to determine the $\lambda_{h}$ and the $P_{k, i}$ 's as follows.

1. Initialize the handoff call arrival rate, $\lambda_{h}^{(0)}=\mu_{h} \lambda_{n} / \mu_{c}$, where the superscript in parentheses means step 0 .

2. Iterate between $P_{k, i}$ 's as in (1)-(8) for all $k$ and $i, N_{u}$ as in (11) and $\lambda_{h}$ as in (12) until

$$
\lambda_{h}^{(n)}-\lambda_{h}^{(n-1)}<\varepsilon_{\lambda_{h}}
$$

where the superscript $n$ in the parentheses denotes the $n$th iteration step, and $\varepsilon_{\lambda_{h}}$ is the error thresholds for $\lambda_{h}$. Using this iterative method, the handoff call arrival rate, $\lambda_{h}$, is obtained iteratively. Note that the initial handoff call arrival rate, $\lambda_{h}^{(0)}$, is set to the approximate handoff call arrival rate under low blocking probabilities as in (13).

\section{Numerical Results}

In this section we present results to examine the performance of new and handoff call blocking probabilities for the MLDGC scheme as well as the performance in system utilization. We consider an MLDGC scheme with 2 levels $(K=2)$. The numerical results for the MLDGC scheme have been obtained by means of the foregoing analysis from Section 4, while those for the FGC scheme have been obtained from the analysis in the Appendix. The parameter values used in the numerical example presented in this section are tabulated in Table 1. The parameter values used are for illustration purposes only.

Table 1. Parameter Values Used

\begin{tabular}{|c|c|c|c|}
\hline & $C=10$ & $C=20$ & $C=30$ \\
\hline $1 / \mu_{c}$ & 1 minute & 1 minute & 1 minute \\
\hline$v$ & $36 \mathrm{~km} / \mathrm{hr}$ & $36 \mathrm{~km} / \mathrm{hr}$ & $36 \mathrm{~km} / \mathrm{hr}$ \\
\hline$s$ & $200 \mathrm{~m}$ & $200 \mathrm{~m}$ & $200 \mathrm{~m}$ \\
\hline $1 / \mu_{h}$ & $1 / 3$ minute & $1 / 3$ minute & $1 / 3$ minute \\
\hline$C_{G 1}$ & 2 & 4 & 6 \\
\hline$C_{G 2}$ & $4,6,8$ & $8,12,16$ & $12,18,24$ \\
\hline$\varepsilon_{\lambda_{h}}$ & $1 \times 10^{-10}$ & $1 \times 10^{-10}$ & $1 \times 10^{-10}$ \\
\hline
\end{tabular}




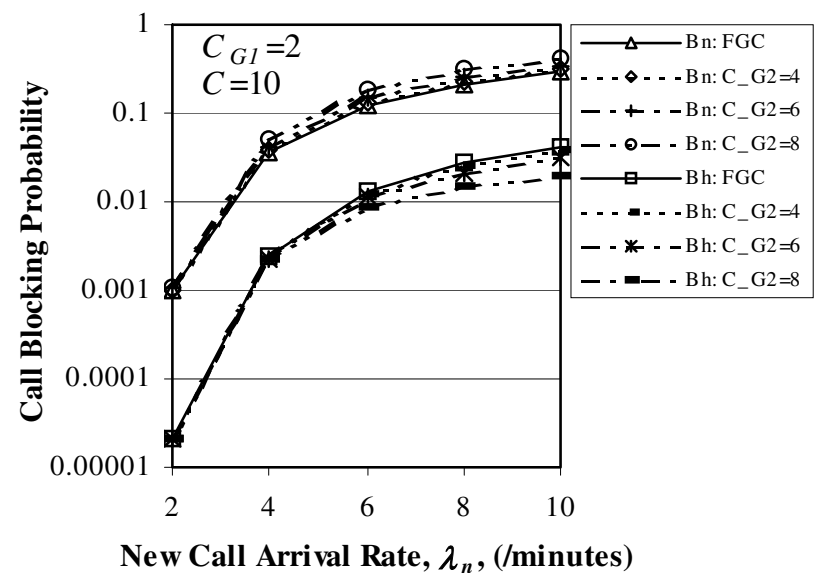

Fig. 2. Call blocking probabilities with $C=10$

The new and handoff call blocking probabilities for the FGC scheme and the proposed MLDGC scheme as a function of the new call arrival rate with $C=10, C=20$ and $C=30$ are shown in Figs. 2, 3 and 4, respectively. For illustration purpose, guard channels $C_{G I}=2, C_{G I}=4$, and $C_{G I}=6$ are chosen for the FGC scheme and level 1 of the MLDGC scheme with $C=10, C=20$, and $C=30$, respectively. For the same purpose, $C_{G 2}=\{4,6,8\}, C_{G 2}=\{8,12,16\}$ and $C_{G 2}=\{12,18,24\}$ are varied for the MLDGC scheme with $C=10, C=20$, and $C=30$, respectively. It is observed that the performances of the new and handoff blocking probabilities in the MLDGC scheme are almost the same as those in the FGC scheme under light load conditions. However, the advantage of the

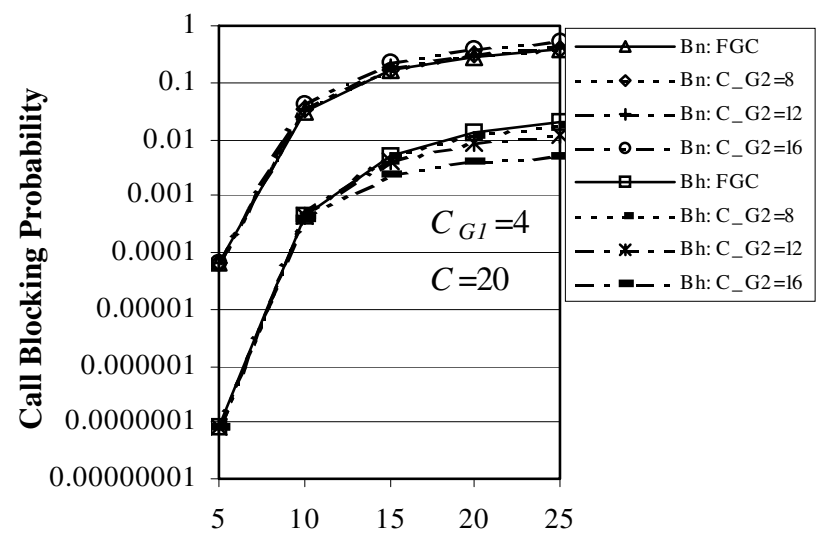

New Call Arrival Rate, $\lambda_{n}$, (/minutes)

Fig. 3. Call blocking probabilities with $C=20$ 


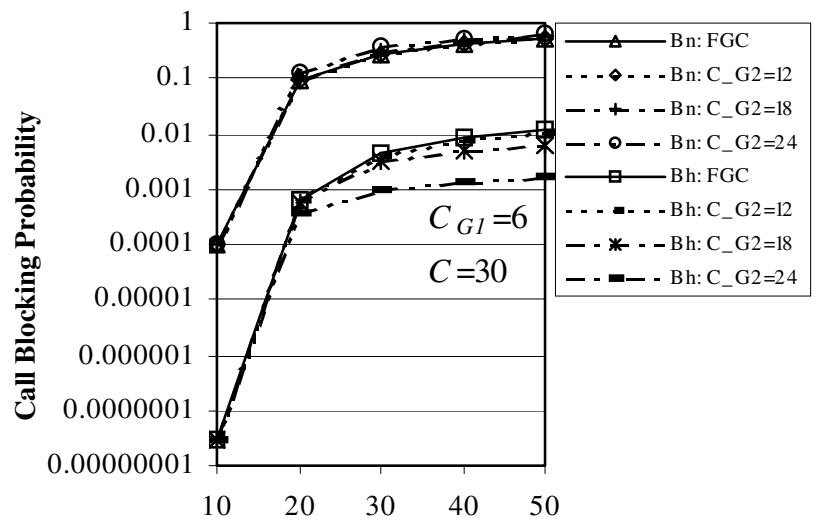

New Call Arrival Rate, $\lambda_{n}$, (/minutes)

Fig. 4. Call blocking probabilities with $C=30$

MLDGC scheme is shown under the heavy load conditions where the performances of the handoff call blocking probabilities of the MLDGC scheme are decreasing lower than those of the FGC scheme with increasing number of guard channels in level 2, $C_{G 2}$, of the MLDGC scheme. These gains in handoff call blocking are obtained at the expense of slight increases of the new call blocking probabilities. Thus the objective of giving higher priority to handoff calls over new calls is further achieved by the MLDGC scheme under heavy load. Hence, extra protection is given to handoff calls during heavy loads.

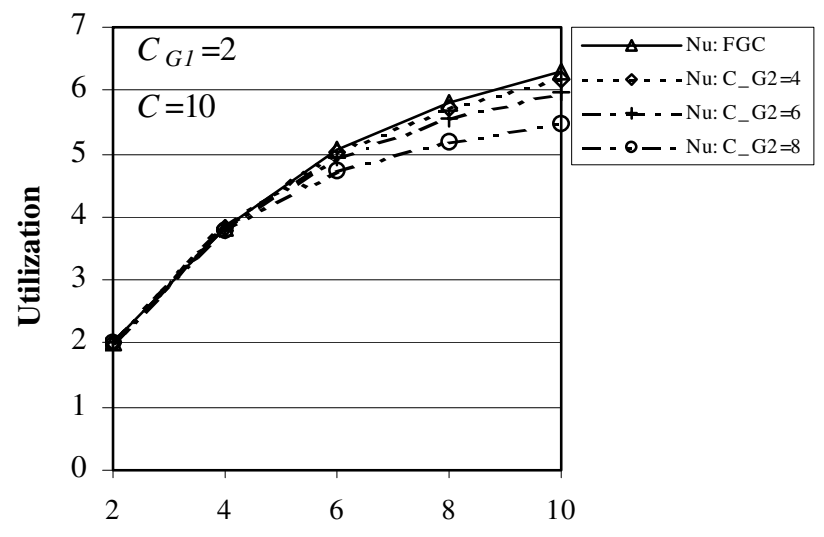

New Call Arrival Rate, $\lambda_{n}$, (/minutes)

Fig. 5. System utilization with $C=10$ 


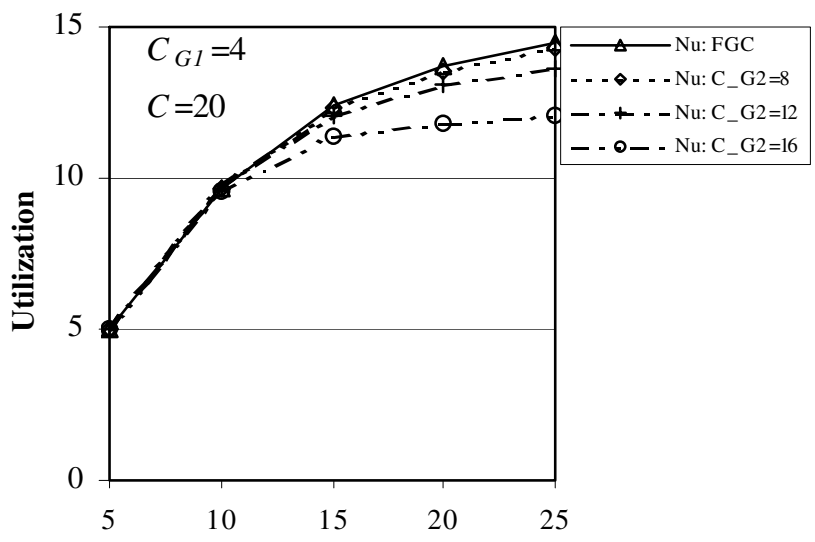

New Call Arrival Rate, $\lambda_{n}$, (/minutes)

Fig. 6. System utilization with $C=20$

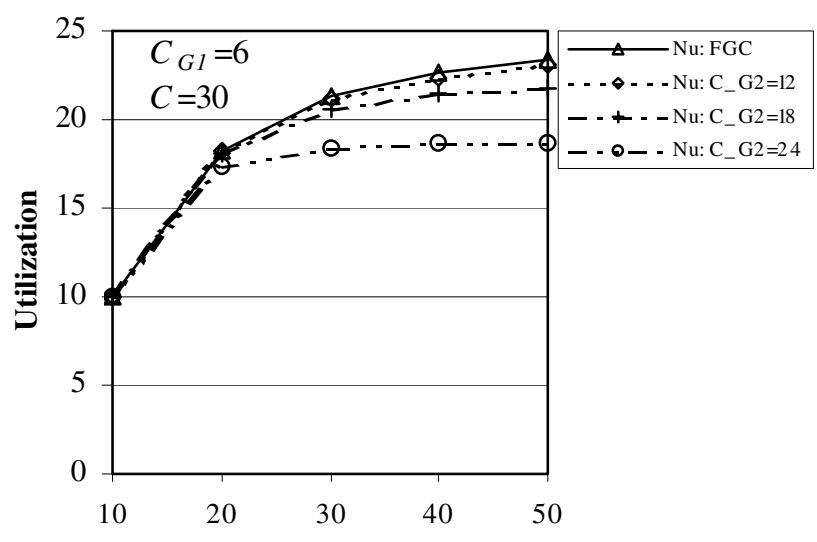

New Call Arrival Rate, $\lambda_{n}$, (/minutes)

Fig. 7. System utilization with $C=30$

Figs. 5, 6 and 7 show the system utilizations for the FGC scheme and the proposed MLDGC scheme as a function of the new call arrival rate for $C=10, C=20$ and $C=30$, respectively. From these figure, it can be seen that the system utilizations for the MLDGC scheme are almost the same as those for the FGC scheme under light load. However, the system utilizations for the MLDGC scheme are lower than those of the FGC scheme under heavy loads. The reason for this is the increased number of guard channels in level 2. Thus this is the tradeoff for using the MLDGC scheme over the FGC scheme. However, as seen earlier in this section, the MLDGC scheme fulfils the 
objective of giving higher priority and protection to handoff calls over new calls under heavy load. Thus such a tradeoff is acceptable.

\section{Concluding Remarks}

A multi-level dynamic guard channels (MLDGC) scheme to enhance access priority for handoff calls in cellular systems is proposed in Section 3. Each additional level has an increasing number of guard channels as triggered by the number of dropped calls. An analytical formulation of the steady state probabilities, new call blocking probability, handoff call blocking probability and system utilization for a proposed multi-level dynamic guard channels scheme with 2 levels is presented in Section 4. The handoff call arrival rate is iteratively obtained. The performance of a 2-level MLDGC scheme with the number of channels in a cell at 10, 20 and 30 are illustrated in numerical examples in Section 5. These examples show that the performance of the MLDGC scheme behaves like a FGC scheme under light load conditions, while the performances of the handoff call blocking probabilities of the MLDGC scheme are decreasing lower than those of the FGC scheme with increasing number of guard channels in level 2 of the MLDGC scheme under heavy load conditions. Thus, the MLDGC scheme introduced in this paper shows promise in terms of fulfilling the objective of giving higher priority and protection to handoff calls over new calls under heavy load, at the expense of a slight degradation in system utilization.

\section{References}

1. Hong, D., Rappaport, S.S.: Traffic Model and Performance Analysis for Cellular Mobile Radio Telephone Systems with Prioritized and Nonprioritized Handoff Procedures - Version 2b. CEAS Tech. Rep. 773, College of Engineering and Applied Sciences, State University of New York, Stony Brook, NY 11794 USA (June 1, 1999)

2. Yu, O.T.W., Leung, V.C.M.: Self-Tuning Prioritized Call Handling Mechanism with Dynamic Guard Channels for Mobile Cellular Systems. IEEE VTC (1996) 1520-1524

3. Yu, O.T.W., Leung, V.C.M.: Adaptive Resource Allocation for Prioritized Call Admission over an ATM-Based Wirelss PCN. IEEE JSAC, Vol. 15, No. 7, (September 1997) 12081225

4. Wei, Y., Lin, C., Ren, F., Raad, R., Dutkiewicz, E.: Dynamic Priority Handoff Scheme in Differentiated QoS Wireless Multimedia Networks. IEEE ISCC (2003) 131-136

5. Lee, S.-H., Park, S.-W.: Handoff with Dynamic Channels in ATM-Based Mobile Networks. International Conference on Information Networking, (1998) 439-442

6. Chen, G.-C., Lee, S.-Y.: Modeling the Static and Dynamic Guard Channel Schemes for Mobile Transactions. International Conference on Parallel and Distributed Computing (1998) 258-265

7. Borst, S.C., Mitra, D.: Virtual partitioning for robust resource sharing: Computational techniques for heterogeneous traffic. IEEE JSAC, Vol. 16, (1998) 668-678 


\section{Appendix - Analysis for a Fixed Guard Channels Scheme}

Let us consider only level 1 in Fig 1 and this is equivalent to a FGC scheme. Let $P_{i}$ be the steady state probabilities in state $i$ of the Markov chain, where $i=0,1, \ldots, C$ and $k=1$ is redundant. Solving this one-dimensional Markov chain by equating probability flows across surfaces of the Markov chain and using the sum of total state probabilities as one, we get

$$
\begin{array}{lr}
P_{i}=P_{0}\left(\frac{\lambda}{\mu}\right)^{i} \frac{1}{i !}, & 1 \leq i \leq C-C_{G 1}, \\
P_{i}=P_{0} \frac{\lambda^{C-C_{G 1}\left(\lambda_{h}\right)^{i-\left(C-C_{G 1}\right)}}}{\mu^{i} i !}, & C-C_{G 1}+1 \leq i \leq C,
\end{array}
$$

where

$$
P_{0}=\left[\sum_{i=0}^{C-C_{G 1}}\left(\frac{\lambda}{\mu}\right)^{i} \frac{1}{i !}+\sum_{i=C-C_{G 1}+1}^{C} \frac{\lambda^{C-C_{G 1}}\left(\lambda_{h}\right)^{i-\left(C-C_{G 1}\right)}}{\mu^{i} i !}\right]^{-1} .
$$

The new call blocking probability, $B_{n}$, is given by

$$
B_{n}=\sum_{i=C-C_{G 1}}^{C} P_{i} .
$$

The handoff call blocking probability, $B_{h}$, is given by

$$
B_{h}=P_{C} .
$$

The system utilization, $N_{u}$, is given by

$$
N_{u}=\sum_{i=1}^{C} i P_{i}=\frac{\lambda_{n}\left(1-B_{n}\right)+\lambda_{h}\left(1-B_{h}\right)}{\mu} .
$$

The handoff call arrival rate is iteratively obtained as in Section 4. 